\title{
Inconsistent Emotion Recognition Deficits across Stimulus Modalities in Huntington's Disease
}

Elin M. Rees ${ }^{1}$, Ruth Farmer ${ }^{2}$, James H. Cole ${ }^{1}$, Susie M.D. Henley ${ }^{3}$, Reiner Sprengelmeyer ${ }^{4}$, Chris Frost $^{2}$, Rachael I. Scahill ${ }^{1}$, Nicola Z. Hobbs ${ }^{1}$ \& Sarah J. Tabrizi ${ }^{1}$

${ }^{1}$ c/o Box 104, University College London, Institute of Neurology, Queen Square, London, UK, WC1N 3BG

elin.rees@ucl.ac.uk (corresponding author)-(+44) 02072420038

james.cole@imperial.ac.uk

r.scahill@ucl.ac.uk

n.hobbs@ucl.ac.uk

s.tabrizi@prion.ucl.ac.uk (corresponding author)

${ }^{2}$ London School of Hygiene and Tropical Medicine, Keppel St, London, UK, WC1E 7HT

ruth.farmer@Ishtm.ac.uk

Chris.Frost@Ishtm.ac.uk

${ }^{3}$ Dementia Research Centre, Department of Neurodegenerative Disease, UCL Institute of Neurology, First floor, 811 Queen Square, London, UK, WC1N 3AR

susie.henley@ucl.ac.uk

${ }^{4}$ School of Psychology and Neurosciences, University of St Andrews, St Mary's Quad, St Andrews, UK, KY16 9JP and Department of Neurology, University of Ulm, Oberer Eselsberg 45,

D-89081, Ulm, Germany

rhs3@st-andrews.ac.uk

Conflict of Interest: Nothing to declare. 


\section{Abstract}

Background: Recognition of negative emotions is impaired in Huntington's Disease (HD). It is unclear whether these emotion-specific problems are driven by dissociable cognitive deficits, emotion complexity, test cue difficulty, or visuoperceptual impairments. This study set out to further characterise emotion recognition in HD by comparing patterns of deficits across stimulus modalities; notably including for the first time in HD, the more ecologically and clinically relevant modality of film clips portraying dynamic facial expressions.

Methods: 15 early HD and 17 control participants were tested on emotion recognition from static facial photographs, non-verbal vocal expressions and one second dynamic film clips, all depicting different emotions.

Results: Statistical evidence of impairment of anger, disgust and fear recognition was seen in HD participants compared with healthy controls across multiple stimulus modalities. The extent of the impairment, as measured by the difference in the number of errors made between HD participants and controls, differed according to the combination of emotion and modality ( $p=0.013$, interaction test). The largest between-group difference was seen in the recognition of anger from film clips.

Conclusions: Consistent with previous reports, anger, disgust and fear were the most poorly recognised emotions by the HD group. This impairment did not appear to be due to task demands or expression complexity as the pattern of between-group differences did not correspond to the pattern of errors made by either group; implicating emotionspecific cognitive processing pathology. There was however evidence that the extent of emotion recognition deficits significantly differed between stimulus modalities. The implications in terms of designing future tests of emotion recognition and care giving are discussed.

Keywords: Huntington's Disease; emotion 


\subsection{Introduction}

Recognising emotions in others from their facial or vocal expressions and body language is a key social skill which is impaired in Huntington's Disease (HD); the literature on which has been reviewed by Henley et al. (Henley et al., 2012). HD is a neurodegenerative, inherited disease caused by an abnormal CAG repeat expansion (Vonsattel \& DiFiglia, 1998). A progressive motor, cognitive and psychiatric phenotype typically manifests at $40-50$ years of age (Novak \& Tabrizi, 2011). Prior to clinical diagnosis (based on motor signs) subtle symptoms of disease are present, including emotion recognition deficits (Tabrizi et al., 2009).

Impairment in this ability could lead to problems with social relationships and therefore a clearer understanding of the profile of this deficit may help guide clinical care. Additionally, a large observational study found recognition of negative emotions to be the only cognitive task in a comprehensive battery to be associated with disease progression in pre-manifest HD (Tabrizi et al., 2013) implicating this as a strong cognitive test to track the pathological development of disease. Investigation of emotion recognition deficits in HD may also help to clarify whether emotion recognition can be conceptualised as a single unified ability or whether there are subdivisions within this, either between emotions or stimulus modalities. For example, if someone can recognise one emotion very well can they recognise all emotions well? If someone is sensitive to emotion portrayed verbally are they also sensitive to visual displays of emotion?

There are six basic emotions, as outlined in much of the seminal work by Paul Ekman and colleagues (Ekman, 1992): happiness, sadness, fear, surprise, anger and disgust. These six are thought to be cross-cultural, with a biological basis. Studies in healthy populations have found a low correlation between a person's ability to recognise positive and negative emotions, leading to the suggestion that these skills may be independent (Suzuki et al., 2014). Similar theories have proposed that emotion recognition may be a broad ability consisting of related, but partially dissociable skills involved in the recognition of positive and negative emotions (Hall, 2001; Schlegel et al., 2012).

Studies in HD initially reported a disproportionate impairment of disgust recognition (Hayes et al., 2007; Wang et al., 2003; Sprengelmeyer et al., 1996). Later findings however suggested that other negative emotions (anger and fear) were equally, if not more affected (deGelder et al., 2008; Hayes et al., 2009; Henley et al., 2008; Milders et al., 2003; Montagne et al., 2006; Snowden et al., 2008; Ille et al., 2011; Tabrizi et al., 2009; Calder et al., 2010). These results are supportive of the concept of dissociable abilities in recognition of positive and negative emotions.

It is unclear in the general population whether emotion recognition from different stimulus modalities can be explained by a unitary ability. There is some evidence that emotion recognition may be modality-specific as performance in a healthy cohort correlated poorly between tests in different modalities (Scherer \& Scherer, 2011). Conversely, Schlegel et al. (Schlegel et al., 2012) concluded that emotion recognition performance in a healthy cohort across stimulus modalities (audio, film, photo and audio-film) could be explained by a single ability dimension. There is therefore no firm agreement on this point. 
In HD, impairments in disgust (Hayes et al., 2007), anger and fear (Snowden et al., 2008; Calder et al., 2010) recognition have been reported across both facial and vocal modalities. The pattern of the severity of emotionspecific deficits across these stimulus types however is unclear. Evidence from vocal stimuli suggests a different deficit to that reported from static facial stimuli, although this relationship was not directly tested (Robotham et al., 2011).

The profile of emotion recognition deficits across different stimulus modalities has important methodological implications. If a varying pattern of impairment is seen across stimulus modalities the use of one to reflect psychosocial functioning would not be appropriate or reflective of the deficit as a whole. If the deficit is statistically similar across stimulus modalities this would implicate a central unified emotion-specific, or negative emotionspecific, pathology underlying multi-modal recognition of that or those emotions.

Overall, the literature suggests that emotion recognition ability can be subdivided into that for positive and negative emotions. It seems that only negative emotion recognition is impaired in HD. It is however unclear whether emotionspecific recognition is consistent across stimulus modalities. The aim of this study was to directly compare, for the first time, the profile of HD-related emotion recognition deficits across different stimulus modalities (facial photos, vocal expressions and dynamic film clips of facial expressions). To our knowledge this is also the first study to investigate emotion recognition in HD from dynamic facial film stimuli, with the aim to establish a more ecologically and clinically relevant profile of this impairment. Finally, in order to test whether the deficits are related to task demands, the HD group's performance was compared to the performance of a matched control group. The pattern of errors made across emotion cues and stimulus modalities was compared to the between-group differences to identify whether the most misidentified emotions were also the ones showing the largest between-group differences.

\subsection{Methods}

\subsubsection{Participants}

Participants were taken from those recruited at the London site of the larger, longitudinal PADDINGTON Study (Hobbs et al., 2013). HD participants ( $N=15$ ) were in stage I of disease based on their Total Functional Capacity (TFC) score from the Unified HD Rating Scale (UHDRS (Huntington Study Group, 1996)), with an average disease burden score (Penney et al., 1997) of 368.61. Fifteen of the 18 control participants were gene-negative siblings or spouses of the HD participants. This was deemed to be preferable to using unrelated healthy controls in order to attempt to match for social and environmental factors. Participant characteristics are summarized in Table 1. One control participant did not complete the BFRT due to time constraints. 


\begin{tabular}{|c|c|c|c|c|}
\hline & \multicolumn{2}{|r|}{ Controls } & \multicolumn{2}{|r|}{ HD } \\
\hline & $\mathbf{N}$ & $\begin{array}{l}\text { Mean (SD) } \\
\text { min - max }\end{array}$ & $\mathbf{N}$ & $\begin{array}{l}\text { Mean (SD) } \\
\min -\max \end{array}$ \\
\hline Age (years) & 18 & $\begin{array}{l}56.31(8.92) \\
43.74-77.71\end{array}$ & 15 & $\begin{array}{l}52.29(9.41) \\
41.16-68.03\end{array}$ \\
\hline Gender (M/F) & $11 / 7$ & & $3 / 12$ & \\
\hline CAG repeat length & & N/A & 15 & $\begin{array}{l}42.87(2.56) \\
39-46\end{array}$ \\
\hline UHDRS: Total motor score & & N/A & 15 & $\begin{array}{l}19.60(10.10) \\
7-45\end{array}$ \\
\hline Disease Burden $^{a}$ & & N/A & 15 & $\begin{array}{l}368.61(103.94) \\
232.60-562.98\end{array}$ \\
\hline Motor response time $(\mathrm{ms})^{b}$ & 18 & $\begin{array}{l}1021.74(182.70) \\
792.18-1487.88\end{array}$ & 15 & $\begin{array}{l}1368.22(313.23) \\
952.41-2021.18\end{array}$ \\
\hline $\begin{array}{l}\text { Benton Facial Recognition Test } \\
\text { (BFRT) score }{ }^{c}\end{array}$ & 17 & $\begin{array}{l}48.94(3.33) \\
43-57\end{array}$ & 15 & $\begin{array}{l}43(4.61) \\
36-51\end{array}$ \\
\hline Education level (ISCED) & 18 & $\begin{array}{l}3.88(1.40) \\
1-6\end{array}$ & 15 & $\begin{array}{l}3.27(1.22) \\
2-5\end{array}$ \\
\hline
\end{tabular}

${ }^{a}$ Disease burden formula (Penney et al., 1997): (CAG-35.5) $\times$ age; ${ }^{b}$ motor response time was assessed and used as a covariate in later analyses to remove a potentially confounding motor component from the main task, the methods for which are described in the section 1.2.2; ${ }^{\mathrm{B}} \mathrm{BFRT}$ (Benton, 1980) score adjusted for age and years of education. ISCED = International Standard Classification of Education.

\subsubsection{Emotion Recognition Task}

The following emotion recognition task was specifically developed for this study. Three sections presented different emotion stimuli:

1. Static black-and-white photos (Manchester Face Set (Whittaker et al., 2001; Snowden et al., 2008))

2. Non-verbal vocal audio clips (Sauter et al., 2010b; Sauter et al., 2010a)

3. One-second film stimuli (Simon et al., 2008).

The Manchester Face Set (Snowden et al., 2008; Whittaker et al., 2001) is a modern variation on the widely-used Ekman face stimuli. The faces were all full-face frontal views of actors posing for a photograph. The vocal stimuli were taken from an emotion sounds test of recorded non-verbal sounds corresponding to happiness (laughter), sadness (sobbing), anger (growls), fear (screams), disgust (retching) and surprise (gasping) (Sauter et al., 2010a; Sauter et al., 2010b). The film stimuli were one-second colour film clips of drama students who were asked to produce each expression in about one second starting with a neutral face and ending at the peak of the expression (Simon et al., 2008). After the one second expression these film clips remained on the screen but static. In a previous study it was noted that 'disgust' in English can have both visceral and moral connotations (Snowden et al., 2008) and therefore variations in meaning might underlie performance differences for 'disgust' across studies involving different languages. Consequently, for consistency and comparability, all disgust stimuli in the current study were visceral in type. 
A forced-choice paradigm was used, instructing the participant to choose the emotion portrayed via a keyboard button press. Each cue was presented or played on a computer screen along with a list of possible emotion options: 1 =anger, 2 =disgust, $3=$ fear, $4=$ happiness, $5=$ sadness, $6=$ surprise. There were no time limits and only when a response was given did the task move to the next stimulus. The stimuli were presented one at a time in a pseudo-random order. Within each stimulus modality the six emotions were presented five times each, resulting in a total of 30 stimuli per stimulus modality and each emotion tested 15 times (across three modalities). The maximum score for the whole task therefore was 90.

Two additional control tests were performed: firstly, the Benton Facial Recognition Test (BFRT short-form (Benton, 1980); a face-matching task) was administered in order to control for basic facial processing ability; secondly, a short motor control task was run. For this, one of the numbers 1-6 appeared on screen in a pseudo-random order and, using the same keyboard buttons as the main task, the participant was asked to press the corresponding button as quickly as they could. Twelve numbers were presented in total. The average time (ms) taken for the button response, excluding the first number, was calculated and used as a control (referred to as motor response time) for motor impairments in statistical analyses.

\subsubsection{Statistical Analysis}

All statistical analyses were conducted in STATA v12. The BFRT was analysed using generalised least squares regression, allowing for different variances in controls and HD participants and adjusting for age, gender, education level and motor response time. Linear regression, with modifications to allow for the non-continuous and nonindependent nature of the outcome variable, was used to analyse the data from the emotion recognition task. The outcome variable in the model was the number of errors (out of five) for each stimulus modality-emotion combination (e.g. photos of fear, film clips of anger etc.). Predictors were a three-way interaction between disease group, stimulus modality and emotion, with adjustment for age, gender, BFRT score, education level and motor response time. To simultaneously allow for the non-normality of the outcome, and for the correlation between the 18 scores for each subject, non-parametric bias corrected $95 \%$ and $99 \%$ bootstrap confidence intervals (Cls) (Efron \& Tibshirani, 1993) were computed from 2000 bootstrap samples, clustered by subject and stratified by group. As such, $p$-value accuracy was reported to $>$ or $<0.05$ or $<0.01$. For the same reason, the three-way interaction was tested with a permutation test. Disease status (control or HD) was permuted 10,000 times, with the Wald test statistic for the interaction term recorded each time. The proportion of times the test statistic was more extreme than the observed statistic from the model was then computed to give the $p$-value.

\subsection{Results}

\subsubsection{Facial Recognition}

Despite the HD group mean of 43 on the BFRT being within the performance range classified as 'normal' (41-54), this was, on average, significantly worse than controls (estimate $-6.76(95 \% \mathrm{Cl}-9.80,-3.71) ; \mathrm{p}<0.001)$. A negative association between BFRT score and emotion recognition errors was also observed. This supported our decision to 
adjust for BFRT scores in the main analyses since we wished to exclude the possibility that any between-group differences in emotion recognition merely reflected differences in facial recognition.

\subsubsection{Emotion Recognition}

\begin{tabular}{|c|c|c|c|c|c|c|c|c|}
\hline \multirow[t]{2}{*}{ Group } & \multirow{2}{*}{$\begin{array}{l}\text { Stimulus } \\
\text { Modality }\end{array}$} & \multicolumn{7}{|c|}{ Mean Number of Errors (SD) } \\
\hline & & Fear & Surprise & Sadness & Anger & Happiness & Disgust & Total \\
\hline \multirow[t]{4}{*}{ Control } & All & $4.56(2.96)$ & $3.94(1.86)$ & $2.94(1.83)$ & $2.44(1.54)$ & $1.56(1.25)$ & $0.72(0.83)$ & $16.17(5.75)$ \\
\hline & Photo & $1.72(1.18)$ & $1.94(1.61)$ & $0.61(0.61)$ & $0.94(0.94)$ & $0.44(0.62)$ & $0.33(0.49)$ & $6.00(3.03)$ \\
\hline & Vocal & $1.33(1.33)$ & $1.28(1.23)$ & $1.67(0.97)$ & $1.39(0.98)$ & $1.06(1.21)$ & $0.22(0.43)$ & $6.94(3.10)$ \\
\hline & Film & $1.50(1.15)$ & $0.72(0.75)$ & $0.67(0.97)$ & $0.11(0.32)$ & $0.06(0.24)$ & $0.17(0.38)$ & $3.22(1.52)$ \\
\hline \multirow[t]{4}{*}{ HD } & All & $8.67(2.94)$ & $5.87(1.73)$ & $5.07(2.76)$ & $7.87(3.89)$ & $2.87(1.68)$ & $4.93(3.39)$ & $39.87(20.04)$ \\
\hline & Photo & $3.20(1.21)$ & $2.47(1.25)$ & $1.60(1.40)$ & $2.80(1.57)$ & $0.73(0.88)$ & $1.73(1.49)$ & $12.53(4.56)$ \\
\hline & Vocal & $3.07(1.33)$ & $2.60(1.40)$ & $2.40(1.12)$ & $2.40(1.30)$ & $1.93(1.58)$ & $1.53(1.25)$ & $13.93(4.83)$ \\
\hline & Film & $2.40(1.24)$ & $0.80(0.77)$ & $1.53(1.41)$ & $2.67(1.84)$ & $0.20(0.56)$ & $1.67(1.40)$ & $9.27(4.27)$ \\
\hline
\end{tabular}

The observed means of the number of errors are presented in Table 2, stratified by group, emotion and modality. Of all six emotions tested, fear was the most misidentified whilst happiness and disgust were recognised most easily in both the control and HD groups. Results of the permutation test showed that the three-way interaction between group, modality and emotion was statistically significant at the $5 \%$ level $(p=0.013)$ and as such the adjusted betweengroup differences in expected number of emotion recognition errors are presented separately for each emotion modality combination in Figure 1 (and Supplementary Table 1). 


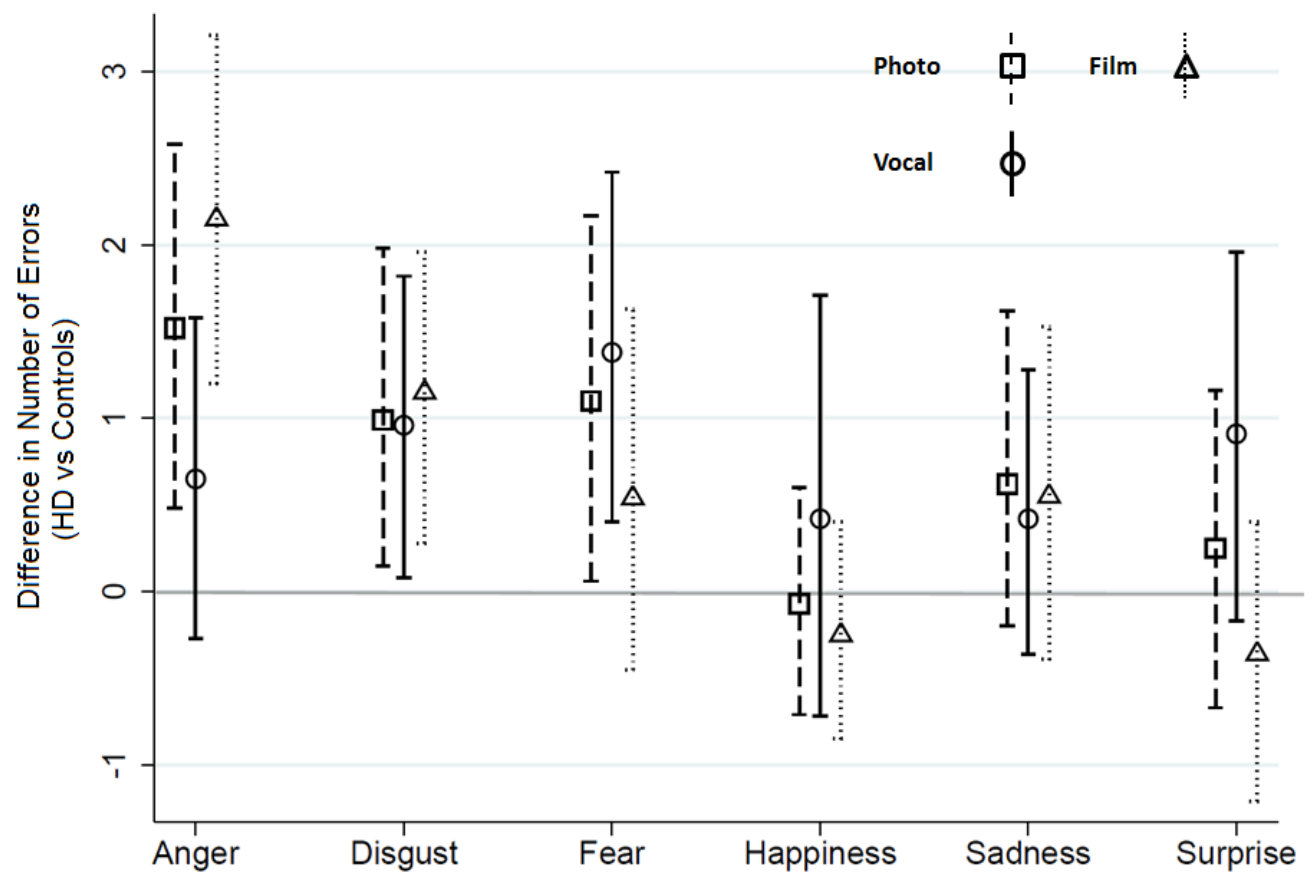

Figure 1. Estimated between-group differences (HD vs Control) in the number of errors out of five for each emotion modality combination, with 95\% bootstrapped bias corrected and accelerated confidence intervals. Positive values indicate that HD participants made more errors than controls. Statistical significance is highlighted with: ${ }^{*} p<0.05 ;{ }^{*} p<0.01$.

For photos, the largest difference between HD and control group performances was seen for anger, with those for fear and disgust also achieving statistical significance. For vocal stimuli the largest difference between HD and control group performances was for fear with the difference for disgust also statistically significant. The difference for anger favoured controls but was smaller than that for photos and film although the confidence interval was relatively wide. For film, as with photos, the largest difference was seen for anger with that for disgust also achieving statistical significance. Here the between-group difference for fear was smaller than seen with the other stimulus modalities although again the difference still favoured controls with the confidence interval being reasonably wide. For happiness, sadness and surprise none of the differences between the HD and control groups achieved statistical significance although in some cases differences were relatively large, albeit with wide confidence intervals.

Of the errors in the HD group the most common mistakes (reported as a percentage of the total number of responses for the emotion displayed) were: anger mistaken for disgust (25\%, i.e. $25 \%$ of the responses made by the HD group to anger stimuli involved the incorrect labelling of these as disgust); disgust mistaken for sadness (10.3\%); fear mistaken for surprise (30.9\%); happiness mistaken for surprise (11.1\%); sadness mistaken for disgust (12.4\%); surprise mistaken for happiness (16.1\%). The percentages of each response made within each stimulus modality are illustrated in Table 3. From this we can see that the results are suggestive of two groupings within which the majority of misidentifications occur in this HD cohort: 1) anger, disgust and sadness; 2) fear, happiness and surprise. 


\begin{tabular}{|c|c|c|c|c|c|c|c|c|c|c|c|c|c|c|c|c|c|c|c|}
\hline \multirow{3}{*}{\multicolumn{2}{|c|}{ Modality }} & \multicolumn{18}{|c|}{ Emotion Displayed } \\
\hline & & \multicolumn{3}{|c|}{ Anger } & \multicolumn{3}{|c|}{ Disgust } & \multicolumn{3}{|c|}{ Fear } & \multicolumn{3}{|c|}{ Happiness } & \multicolumn{3}{|c|}{ Sadness } & \multicolumn{3}{|c|}{ Surprise } \\
\hline & & Photo & Vocal & Film & Photo & Vocal & Film & Photo & Vocal & Film & Photo & Vocal & Film & Photo & Vocal & Film & Photo & Vocal & Film \\
\hline \multirow{6}{*}{ 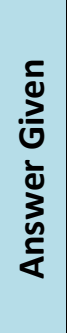 } & Anger & $44.0 \%$ & $51.4 \%$ & $46.7 \%$ & $9.3 \%$ & $6.8 \%$ & $10.7 \%$ & $5.3 \%$ & $9.5 \%$ & $8.0 \%$ & $1.3 \%$ & $1.3 \%$ & $0.0 \%$ & $5.3 \%$ & $8.0 \%$ & $4.0 \%$ & $2.7 \%$ & $2.7 \%$ & $4.0 \%$ \\
\hline & Disgust & $18.7 \%$ & $13.5 \%$ & $42.7 \%$ & $65.3 \%$ & $68.9 \%$ & $66.7 \%$ & $9.3 \%$ & $14.9 \%$ & $10.7 \%$ & $0.0 \%$ & $1.3 \%$ & $0.0 \%$ & $10.7 \%$ & $10.7 \%$ & $16.0 \%$ & $8.0 \%$ & $13.5 \%$ & $2.7 \%$ \\
\hline & Fear & $5.3 \%$ & $16.2 \%$ & $4.0 \%$ & $4.0 \%$ & $5.4 \%$ & $5.3 \%$ & $36.0 \%$ & $39.2 \%$ & $52.0 \%$ & $1.3 \%$ & $2.7 \%$ & $1.3 \%$ & $4.0 \%$ & $5.3 \%$ & $8.0 \%$ & $5.3 \%$ & $9.5 \%$ & $9.3 \%$ \\
\hline & Happiness & $1.3 \%$ & $2.7 \%$ & $0.0 \%$ & $5.3 \%$ & $4.1 \%$ & $1.3 \%$ & $0.0 \%$ & $1.4 \%$ & $0.0 \%$ & $85.3 \%$ & $61.3 \%$ & $96.0 \%$ & $4.0 \%$ & $18.7 \%$ & $1.3 \%$ & $25.3 \%$ & $23.0 \%$ & $0.0 \%$ \\
\hline & Sadness & $21.3 \%$ & $10.8 \%$ & $4.0 \%$ & $10.7 \%$ & $6.8 \%$ & $13.3 \%$ & $5.3 \%$ & $14.9 \%$ & $1.3 \%$ & $0.0 \%$ & $12.0 \%$ & $2.7 \%$ & $68.0 \%$ & $52.0 \%$ & $69.3 \%$ & $8.0 \%$ & $2.7 \%$ & $0.0 \%$ \\
\hline & Surprise & $9.3 \%$ & $5.4 \%$ & $2.7 \%$ & $5.3 \%$ & $8.1 \%$ & $2.7 \%$ & $44.0 \%$ & $20.3 \%$ & $28.0 \%$ & $12.0 \%$ & $21.3 \%$ & $0.0 \%$ & $8.0 \%$ & $5.3 \%$ & $1.3 \%$ & $50.7 \%$ & $48.6 \%$ & $84.0 \%$ \\
\hline
\end{tabular}




\subsection{Discussion}

This was the first study to compare the profile of the emotion recognition deficit in HD across different stimulus modalities, including the first use of dynamic film stimuli of facial expressions. It is thought that recognition of emotions of positive and negative valence constitutes dissociable abilities but it is currently unclear whether emotion recognition across different stimulus modalities should be conceptualised as a unitary ability. The consistent finding of impaired negative emotion recognition in $\mathrm{HD}$ is supportive of the theory of positive and negative subdimensions in emotion recognition ability. This deficit appears to be independent of individual stimulus or task demands as the pattern of errors made did not match the pattern of HD-related deficit in comparison to the control group performance. This valence-specific emotion recognition ability was found in this study to be consistent across stimulus modalities but the emotion-specific profile of the impairment differed significantly. This could be explained by the theory of related yet specific skills that incrementally contribute to emotion recognition ability (Hall, 2001; Schlegel et al., 2012). These findings show that the use of emotion recognition performance from one stimulus modality is not representative of the deficit as a whole and therefore is not fully reflective of psychosocial functioning.

There does not seem to be a clear link between the number of errors and the pattern of impairment in HD participants compared with controls. For example, disgust was the most accurately identified emotion by the control group and the second best only behind happiness in the HD group, yet recognition of this emotion was consistently different between groups in all stimulus modalities. These findings do not support the theory that the disproportionate emotion-specific deficits found in HD are a result of increased cognitive demand, test cue difficulty or expression complexity but instead are indicative of emotion-specific or valence-specific emotion recognition cognitive pathology.

The significant three-way interaction between disease group, stimulus modality and emotion suggests however that impairment may not be entirely consistent across stimulus modality and emotion. The largest between-modality difference was shown between film and vocal anger recognition; anger being recognised by the HD group at a closer level to controls from the vocal cues than the film clips. In addition impairment in fear recognition was estimated to be greater than that for anger in the vocal stimuli, whereas the converse was true for film stimuli. If these observed between-modality differences in emotion recognition deficits in HD were to be replicated in a larger sample, this finding could have implications in terms of designing future tests and care giving. For example, emotion recognition performance from the traditional static photo modality does not 
appear to be as clinically relevant as previously assumed; in order to establish a more accurate profile of this impairment in everyday life a multi-modal test of emotion recognition is recommended. In terms of care giving these results suggest that educating care-givers of this potential deficit and training in the use of a varied communication style, incorporating both visual and vocal expressions, may facilitate emotion recognition and consequently social functioning.

From the mistakes being made we can see that there are two groupings of emotions that are being confused in HD: 1) anger, disgust and sadness; 2) fear, happiness and surprise. These groupings are consistent with the schematic representations of the relations between different facial expression proposed by Woodworth \& Scholsberg (Woodworth \& Schlosberg, 1954) and Calder et al.'s (Calder et al., 1996) hexagonal continuum of emotions: happiness to surprise to fear to sadness to disgust to anger to happiness. The two groupings can be thought of as open and closed expressions: with anger, disgust and sadness characterised by a closing of the eyes and lowering of the eyebrows; and fear, happiness and surprise characterised by an opening of the eyes and raising of the eyebrows. These similarities between expressions increase the demands on perceptual processing when discriminating between emotions within a 'set'. In terms of complexity, disgust and anger also have a conceptual overlap and this may be a factor behind anger-disgust mistakes.

In the HD group the error rates in the photo, vocal and film stimuli were $41.8 \%, 46.4 \%$ and $30.9 \%$ respectively. Emotion recognition from film cues (the least effected modality) may be more reflective of typical social interactions than static photos or vocal cues and therefore performance here is arguably of more clinical relevance. Although disgust, anger and fear recognition combined across stimulus modalities was significantly impaired in the HD group compared with control group performance, these responses were erroneous just $32.9 \%, 52.5 \%$ and $57.8 \%$ of the time respectively; levels indicative of substantial remaining functional capacity in this early-stage HD cohort.

Previous studies of gene-negative siblings or spouses of HD participants have found impairments in facial anger recognition compared with unrelated healthy controls (Gray et al., 1997; Sprengelmeyer et al., 2006). Our control group (15 of the 18 were gene-negative siblings or spouses of HD participants) found anger to be only the fourth (out of six) most difficult emotion to recognise when errors from all stimulus modalities were combined (2.44 mean errors (SD 1.54) out of 15 responses). This does not support the idea of anger recognition impairments in gene-negative people from an HD family although this was not done in comparison to an unrelated healthy control group.

This study was limited by sample size and the exploratory findings would clearly benefit from replication. Due to the clinical population available to us there was an imbalance in males and 
females within the two groups. This was accounted for in all statistical analyses by adjusting for gender. The fact that the behavioural results corroborate previous work suggests that this sample is likely to be representative and that these novel, albeit tentative, findings are worthy of follow-up.

In conclusion, the direct comparison of emotion recognition deficits across multiple stimulus modalities make this study a thorough investigation of several aspects of emotion recognition in HD. Consistent with previous reports, anger, disgust and fear recognition was shown to be impaired in HD. There was however evidence of differences in the extent of impairment relative to controls for specific emotions between the traditional static photo modality, vocal stimuli, and the more ecologically and clinically relevant film clips, never before used in HD. Impairment does not seem to be due to task demands or expression complexity as the pattern of between-group differences did not correspond to the pattern of errors made by either group, therefore implicating emotion-specific cognitive processing pathology. These findings may have implications for future test design and care giving. 


\section{Acknowledgements}

The authors would like to thank the participants who took part in this study along with all the Work Package 2 site staff and Dr Sarah Gregory for her helpful comments and suggestions.

This study has been supported by the European Union - PADDINGTON project, contract n. HEALTHF2-2010-261358. Work was undertaken at UCL and the Dementia Research Centre (DRC) and supported by the NIHR Queen Square Dementia BRU. UCL receives a proportion of funding from the Department of Health's NIHR Biomedical Research Centres funding scheme and CHDI/High Q Foundation, a non-for-profit organization dedicated to finding treatments for HD. The DRC is an Alzheimer's Research UK Co-ordinating Centre and has also received equipment funded by Alzheimer's Research UK and Brain Research Trust. SJT acknowledges support of the National Institute for Health Research through the Dementias and Neurodegenerative Research Network, DeNDRoN.

Reference List

Benton, A. L. (1980). The Neuropsychology of Facial Recognition. American Psychologist, 35(2), 176186. Retrieved from ISI:A1980JK95600003

Calder, A. J., Keane, J., Young, A. W., Lawrence, A. D., Mason, S., \& Barker, R. A. (2010). The relation between anger and different forms of disgust: implications for emotion recognition impairments in Huntington's disease. Neuropsychologia, 48(9), 2719-2729. doi:S00283932(10)00200-9 [pii];10.1016/j.neuropsychologia.2010.05.019 [doi]. Retrieved from PM:20580641

Calder, A. J., Young, A. W., Rowland, D., Perrett, D. I., Hodges, J. R., \& Etcoff, N. L. (1996). Facial emotion recognition after bilateral amygdala damage: Differentially severe impairment of fear. Cognitive Neuropsychology, 13(5), 699-745. Retrieved from ISI:A1996VE79300005

deGelder, B., Van den Stock, J., Balaguer, R. D., \& Bachoud-Levi, A. C. (2008). Huntington's disease impairs recognition of angry and instrumental body language. Neuropsychologia, 46(1), 369- 
373. doi:S0028-3932(07)00364-8 [pii];10.1016/j.neuropsychologia.2007.10.015 [doi].

Retrieved from PM:18061217

Efron, B., \& Tibshirani, R. J. (1993). An introduction to the bootstrap. New York: Chapman \& Hall.

Ekman, P. (1992). Are There Basic Emotions. Psychological Review, 99(3), 550-553. Retrieved from ISI:A1992JE92400010

Gray, J. M., Young, A. W., Barker, W. A., Curtis, A., \& Gibson, D. (1997). Impaired recognition of disgust in Huntington's disease gene carriers. Brain, 120 ( Pt 11), 2029-2038. Retrieved from PM:9397019

Hall, J. A. (2001). The PONS test and the psychometric approach to measuring interpersonal sensitivity. In J.A. Hall \& F. J. Bernieri (Eds.), Interpersonal Sensitivity: Theory and Measurement (pp. 143-160). Mahwah, NJ: Erlbaum.

Hayes, C. J., Stevenson, R. J., \& Coltheart, M. (2007). Disgust and Huntington's disease. Neuropsychologia, 45(6), 1135-1151. doi:S0028-3932(06)00412-X [pii];10.1016/j.neuropsychologia.2006.10.015 [doi]. Retrieved from PM:17126861

Hayes, C. J., Stevenson, R. J., \& Coltheart, M. (2009). The Processing of Emotion in Patients With Huntington's Disease: Variability and Differential Deficits in Disgust. Cognitive and Behavioral Neurology, 22(4), 249-257. Retrieved from ISI:000272940200007

Henley, S. M., Novak, M. J., Frost, C., King, J., Tabrizi, S. J., \& Warren, J. D. (2012). Emotion recognition in Huntington's disease: a systematic review. Neurosci.Biobehav.Rev., 36(1), 237253. doi:S0149-7634(11)00112-6 [pii];10.1016/j.neubiorev.2011.06.002 [doi]. Retrieved from PM:21699916 
Henley, S. M., Wild, E. J., Hobbs, N. Z., Warren, J. D., Frost, C., Scahill, R. I. et al. (2008). Defective emotion recognition in early HD is neuropsychologically and anatomically generic. Neuropsychologia, 46(8), 2152-2160. doi:S0028-3932(08)00087-0 [pii];10.1016/j.neuropsychologia.2008.02.025 [doi]. Retrieved from PM:18407301

Hobbs, N. Z., Cole, J. H., Farmer, R. E., Rees, E. M., Crawford, H. E., Malone, I. B. et al. (2013). Evaluation of multi-modal, multi-site neuroimaging measures in Huntington's disease: Baseline results from the PADDINGTON study. Neurolmage: Clinical, 2, 204-211.

Huntington Study Group. (1996). Unified Huntington's Disease Rating Scale: reliability and consistency. Huntington Study Group. Mov Disord., 11(2), 136-142. doi:10.1002/mds.870110204 [doi]. Retrieved from PM:8684382

Ille, R., Schafer, A., Scharmuller, W., Enzinger, C., Schoggl, H., Kapfhammer, H. P. et al. (2011). Emotion recognition and experience in Huntington disease: a voxel-based morphometry study. J.Psychiatry Neurosci., 36(6), 383-390. doi:10.1503/jpn.100143 [pii];10.1503/jpn.100143 [doi]. Retrieved from PM:21406159

Milders, M., Crawford, J. R., Lamb, A., \& Simpson, S. A. (2003). Differential deficits in expression recognition in gene-carriers and patients with Huntington's disease. Neuropsychologia, 41(11), 1484-1492. doi:S0028393203000794 [pii]. Retrieved from PM:12849766

Montagne, B., Kessels, R. P., Kammers, M. P., Kingma, E., De Haan, E. H., Roos, R. A. et al. (2006). Perception of emotional facial expressions at different intensities in early-symptomatic Huntington's disease. Eur.Neurol., 55(3), 151-154. doi:93215 [pii];10.1159/000093215 [doi]. Retrieved from PM:16682799 
Novak, M. J. U., \& Tabrizi, S. J. (2011). Huntington'S Disease: Clinical Presentation and Treatment. Pathophysiology, Pharmacology, and Biochemistry of Dyskinesia, 98, 297-323. Retrieved from ISI:000295559600013

Penney, J. B., Vonsattel, J. P., MacDonald, M. E., Gusella, J. F., \& Myers, R. H. (1997). CAG repeat number governs the development rate of pathology in Huntington's disease. Annals of Neurology, 41(5), 689-692. Retrieved from ISI:A1997WZ80900020

Robotham, L., Sauter, D. A., Bachoud-Levi, A. C., \& Trinkler, I. (2011). The impairment of emotion recognition in Huntington's disease extends to positive emotions. Cortex, 47(7), 880-884. doi:S0010-9452(11)00039-6 [pii];10.1016/j.cortex.2011.02.014 [doi]. Retrieved from PM:21429482

Sauter, D. A., Eisner, F., Calder, A. J., \& Scott, S. K. (2010a). Perceptual cues in nonverbal vocal expressions of emotion. Quarterly Journal of Experimental Psychology, 63(11), 2251-2272. Retrieved from ISI:000283684100013

Sauter, D. A., Eisner, F., Ekman, P., \& Scott, S. K. (2010b). Cross-cultural recognition of basic emotions through nonverbal emotional vocalizations. Proc.Natl.Acad.Sci.U.S.A, 107(6), 2408-2412. doi:0908239106 [pii];10.1073/pnas.0908239106 [doi]. Retrieved from PM:20133790

Scherer, K. R., \& Scherer, U. (2011). Assessing the Ability to Recognize Facial and Vocal Expressions of Emotion: Construction and Validation of the Emotion Recognition Index. Journal of Nonverbal Behavior, 35(4), 305-326. Retrieved from ISI:000299944400006

Schlegel, K., Grandjean, D., \& Scherer, K. R. (2012). Emotion recognition: Unidimensional ability or a set of modality- and emotion-specific skills? Personality and Individual Differences, 53(1), 1621. Retrieved from ISI:000303957800003 
Simon, D., Craig, K. D., Gosselin, F., Belin, P., \& Rainville, P. (2008). Recognition and discrimination of prototypical dynamic expressions of pain and emotions. Pain, 135(1-2), 55-64. doi:S03043959(07)00248-5 [pii];10.1016/j.pain.2007.05.008 [doi]. Retrieved from PM:17583430

Snowden, J. S., Austin, N. A., Sembi, S., Thompson, J. C., Craufurd, D., \& Neary, D. (2008). Emotion recognition in Huntington's disease and frontotemporal dementia. Neuropsychologia, 46(11), 2638-2649. doi:S0028-3932(08)00165-6

[pii];10.1016/j.neuropsychologia.2008.04.018 [doi]. Retrieved from PM:18533200

Sprengelmeyer, R., Schroeder, U., Young, A. W., \& Epplen, J. T. (2006). Disgust in pre-clinical Huntington's disease: a longitudinal study. Neuropsychologia, 44(4), 518-533. doi:S00283932(05)00255-1 [pii];10.1016/j.neuropsychologia.2005.07.003 [doi]. Retrieved from PM:16098998

Sprengelmeyer, R., Young, A. W., Calder, A. J., Karnat, A., Lange, H., Homberg, V. et al. (1996). Loss of disgust. Perception of faces and emotions in Huntington's disease. Brain, 119 ( Pt 5), 16471665. Retrieved from PM:8931587

Suzuki, A., Hoshino, T., \& Shigemasu, K. (2014). Happiness is unique: A latent structure of emotion recognition traits revealed by statistical model comparison. Personality and Individual Differences, 48, 196-201.

Tabrizi, S. J., Langbehn, D. R., Leavitt, B. R., Roos, R. A. C., Durr, A., Craufurd, D. et al. (2009). Biological and clinical manifestations of Huntington's disease in the longitudinal TRACK-HD study: cross-sectional analysis of baseline data. Lancet Neurology, 8(9), 791-801. Retrieved from ISI:000269241700007

Tabrizi, S. J., Scahill, R. I., Owen, G., Durr, A., Leavitt, B. R., Roos, R. A. et al. (2013). Predictors of phenotypic progression and disease onset in premanifest and early-stage Huntington's 
disease in the TRACK-HD study: analysis of 36-month observational data. Lancet Neurology, 12(7), 637-649. Retrieved from ISI:000320977100007

Vonsattel, J. P. G., \& DiFiglia, M. (1998). Huntington disease. Journal of Neuropathology and Experimental Neurology, 57(5), 369-384. Retrieved from ISI:000073611400001

Wang, K., Hoosain, R., Yang, R. M., Meng, Y., \& Wang, C. Q. (2003). Impairment of recognition of disgust in Chinese with Huntington's or Wilson's disease. Neuropsychologia, 41(5), 527-537. doi:S0028393202001719 [pii]. Retrieved from PM:12559147

Whittaker, J. F., Deakin, J. F. W., \& Tomenson, B. (2001). Face processing in schizophrenia: defining the deficit. Psychological Medicine, 31(3), 499-507. Retrieved from ISI:000168192700012

Woodworth, R. S., \& Schlosberg, H. (1954). Experimental psychology: revised edition. New York: Henry Holt. 